University of Texas at El Paso

ScholarWorks@UTEP

$12-1999$

\title{
Intervals (Pairs of Fuzzy Values), Triples, etc.: Can We Thus Get an Arbitrary Ordering?
}

Vladik Kreinovich

The University of Texas at El Paso, vladik@utep.edu

Masao Mukaidono

Follow this and additional works at: https://scholarworks.utep.edu/cs_techrep

Part of the Computer Engineering Commons

Comments:

UTEP-CS-99-38a.

Published in Proceedings of the 9th IEEE International Conference on Fuzzy Systems (FUZZIEEE'2000), San Antonio, Texas, May 7-10, 2000, Vol. 1, pp. 234-238.

\section{Recommended Citation}

Kreinovich, Vladik and Mukaidono, Masao, "Intervals (Pairs of Fuzzy Values), Triples, etc.: Can We Thus Get an Arbitrary Ordering?" (1999). Departmental Technical Reports (CS). 577.

https://scholarworks.utep.edu/cs_techrep/577

This Article is brought to you for free and open access by the Computer Science at ScholarWorks@UTEP. It has been accepted for inclusion in Departmental Technical Reports (CS) by an authorized administrator of ScholarWorks@UTEP.For more information, please contact Iweber@utep.edu. 


\title{
Intervals (Pairs of Fuzzy Values), Triples, etc.: Can We Thus Get an Arbitrary Ordering?
}

\author{
Vladik Kreinovich \\ Computer Science, University of Texas, El Paso, TX 79968, USA, vladik@cs.utep.edu \\ Masao Mukaidono \\ Computer Science, Meiji University, Kawasaki-shi 214 Japan, masao@cs.meiji.ac.jp
}

\begin{abstract}
Traditional fuzzy logic uses real numbers as truth values. This description is not always adequate, so in interval-valued fuzzy logic, we use pairs $\left(t^{-}, t^{+}\right)$of real numbers, $t^{-} \leq t^{+}$, to describe a truth value. To make this description even more adequate, instead of using real numbers to described each value $t^{-}$and $t^{+}$, we can use intervals, and thus get fuzzy values which can be described by 4 real numbers each. We can iterate this procedure again and again. The question is: can we get an arbitrary partially ordered set in this manner? An arbitrary lattice? In this paper, we show that although we cannot thus generate arbitrary lattices, we can actually generate an arbitrary partially ordered set in this manner. In this sense, the "intervalization" operation is indeed universal.
\end{abstract}

\section{INTRODUCTION}

\section{A. Traditional Fuzzy Logic and Its Drawbacks}

Traditional fuzzy uses real numbers as truth values. If we use the simplest possible $\&$-operation $\min (a, b)$, then we can describe the relation $a \leq b$ as $a \& b=a$, i.e., as $\min (a, b)=a$. Thus defined relation coincides with the normal ordering on the interval $[0,1]$.

The description provided by the traditional [0,1]-based fuzzy logic is not always adequate: e.g., it does not distinguish between the situations in which we know nothing about a certain statement, and a situation in which we have exactly as many arguments in favor of this statement as we have against it.

\section{B. Interval-Valued Fuzzy Logic}

Interval-valued fuzzy logic enables us to describe this difference. Namely, in interval-valued fuzzy logic, instead of describing a truth value as a single number $t$, we describe the truth value by an interval $\left[t^{-}, t^{+}\right]$of its possible values. In these terms, the situation when we know nothing about a statement can be represented by the interval $[0,1]$, while a situation in which we have an equal amount of arguments in favor of a statement and of its negation can be represented, e.g., as [0.5, 0.5].
In order to describe a natural ordering on the resulting set of truth values, we must describe the corresponding \&operation. If the only thing we know about the "degree of truth" of a statement $A$ is that it belongs to the interval $\mathbf{t}(A)=\left[t^{-}(A), t^{+}(A)\right]$, and the only thing we know about the "degree of truth" of a statement $B$ is that it belongs to the interval $\mathbf{t}(B)=\left[t^{-}(B), t^{+}(B)\right]$, then possible values of $t(A \& B)=\min (t(A), t(B))$ for a set

$$
\begin{gathered}
\left\{t(A) \& t(B) \mid t(A) \in\left[t^{-}(A), t^{+}(A)\right]\right. \text { and } \\
\left.t(B) \in\left[t^{-}(B), t^{+}(B)\right]\right\} .
\end{gathered}
$$

One can show that this set is itself an interval, namely, the interval

$$
\mathbf{t}(A \& B)=\left[\min \left(t^{-}(A), t^{-}(B)\right), \min \left(t^{+}(A), t^{+}(B)\right)\right] .
$$

Therefore, if we define $\mathbf{t}(A) \leq \mathbf{t}(B)$ as meaning that $\mathbf{t}(A \& B)=\mathbf{t}(A)$, then we can conclude that $\mathbf{t}(A) \leq \mathbf{t}(B)$ if and only if $t_{A}^{-} \leq t_{B}^{-}$and $t_{A}^{+} \leq t_{B}^{+}$.

C. One More "Intervalization" Step: From IntervalValued Fuzzy Logic to “4-Valued” Fuzzy Logic and Further

In interval-valued fuzzy logic, we use exact numbers to describe both degrees $t^{-}$and $t^{+}$. In real life, people are reluctant to describe their degrees of certainty by exact numbers. For example, it is difficult to distinguish between the degree of belief 0.7 and 0.71 . A more adequate way of describing each degree of certainty is by using not exact numbers, but intervals of possible values.

Therefore, to describe each degree of certainty, we must use a pair of intervals $\left[t^{--}, t^{-+}\right]$and $\left[t^{+-}, t^{++}\right]$. We must guarantee that whatever numbers we pick from these intervals, we will always have $t^{-} \leq t^{+}$. To guarantee this inequality, we must require that $t^{--} \leq t^{-+} \leq t^{+-} \leq t^{++}$. In other words, instead of two linearly ordered numbers, we have four linearly ordered numbers.

Once can easily see that the natural ordering between such numbers is also component-wise:

$$
\begin{gathered}
\left(t^{--}(A), t^{-+}(A), t^{+-}(A), t^{++}(A)\right) \leq \\
\left(t^{--}(B), t^{-+}(B), t^{+-}(B), t^{++}(B)\right)
\end{gathered}
$$


if and only if $t^{--}(A) \leq t^{--}(B), t^{-+}(A) \leq t^{-+}(B)$, $t^{+-}(A) \leq t^{++}(B)$, and $t^{++}(A) \leq t^{++}(B)$.

To make a description more adequate, we can perform one more intervalization step, etc.; see, e.g., [1]-[6] and references therein.

At each step, we describe each degree of belief by a linearly ordered sequence of real numbers

$$
t_{1} \leq t_{2} \leq \ldots \leq t_{n}
$$

for which $A \leq B$ if and only for each $i, t_{i}(A) \leq t_{i}(B)$.

\section{Formulation of the Problem}

A natural question is: if we repeat this procedure again and again, can we get an arbitrary partial order? In other words, is the intervalization procedure which underlies intuitionistic fuzzy logic, really universal?

In this paper, we show that the answer to this question is "yes", intervalization is indeed universal.

\section{Definitions and the Main Result}

Before we formulate the result in precise terms, let us first give a general definition of what we mean by the result of an intervalization.

- The partial ordered set, interpreted as the set of all truth values, will be denoted by $V$. In the above $n$-dimensional intervalization example, $V$ is the set of all ordered $n$-tuples of real numbers with a component-wise ordering.

- We have the original linearly ordered set $L$; in our example, $L=[0,1]$.

- We also have a linearly ordered set of indices $A$; in the above example, $A=\{1 \leq 2 \leq \ldots \leq n\}$.

- We have a value from $L$ assigned to every truth value $v$ and to every index $a \in A$. In our example, the value assigned to $x=\left(x_{1}, \ldots, x_{n}\right) \in V$ and $i \in A=$ $\{1, \ldots, n\}$ is $x_{i} \in[0,1]=L$. We will denote this value by $f(v, a)$. In precise terms, $f$ is a function from $V \times A$ to $L$.

- The fact that $x_{i}$ is a linearly ordered sequence of values means that for every $v$, if $a \leq b$, then $f(v, a) \leq$ $f(v, b)$.

- We also need to describe the fact that the order on $V$ is defined component-wise. In our notations, this means that $u \leq v$ if and only if for all $a, f(u, a) \leq$ $f(v, a)$.
Now, we are ready for our definition and for the corresponding main result:

Definition 1. We say that a partially ordered set $V$ can be obtained by intervalization if there exist linearly ordered sets $L$ and $A$ and a function $f: V \times A \rightarrow L$ for which the following two conditions hold:

- For every $v \in V, a \in A$, and $b \in A$, if $a \leq b$, then $f(v, a) \leq f(v, b)$.

- For every $u, v \in V, u \leq v$ if and only if

$$
\forall a(f(u, a) \leq f(v, a)) .
$$

Theorem 1. Every partially ordered set can be obtained by intervalization.

Comments.

- For reader's convenience, all the proofs are presented in the last section.

- As we will see from the proof, if $V$ is a finite set, then we can take finite sets $A$ and $L$.

- We can also see from the proof that if the set $V$ is infinite countable, then we can choose, as $L$, a countable linearly ordered set which can be thus embedded into $[0,1]$. In other words, in this case, we can use $L=[0,1]$.

III. First Auxiliary Result: Intervalization Is A Universal ToOl for ORDERs BUt Not For Lattice Structures

The set of truth values is usually described not only by its partial ordering relation, but also by the corresponding lattice operations (which usually correspond to "and" and "or"). We already know that the partial order can be obtained by intervalization; a natural next question is: can we obtain the lattice structure in the same way? This time, the answer is negative:

Definition 2. We say that a lattice $V$ with operations $\checkmark$ and $\wedge$ can be obtained by intervalization if there exist linearly ordered sets $L$ and $A$ and a function $f: V \times A \rightarrow L$ for which the following three conditions hold:

- For every $v \in V, a \in A$, and $b \in A$, if $a \leq b$, then $f(v, a) \leq f(v, b)$.

- For every $u, v \in V, u \leq v$ if and only if

$$
\forall a(f(u, a) \leq f(v, a)) .
$$

- For every $u, v \in V$ and for every $a \in A$,

$$
\begin{gathered}
f(u \vee v, a)=\max (f(u, a), f(v, a)) \text { and } \\
f(u \wedge v, a)=\min (f(u, a), f(v, a)) .
\end{gathered}
$$


Theorem 2. Not every lattice can be obtained by intervalization.

\section{Second Auxiliary Result: What Will Happen If We Apply Intervalization to Classical 2-Valued Logic?}

The next natural question is as follows: we started intervalization with a (generalized) fuzzy set, i.e., with a linearly ordered set $L$. What if we start instead with a set $L_{0}=\{0<1\}$ of truth values of classical 2 -valued logic?

Definition 3. We say that a partial ordered set $V$ can be obtained by intervalization of classical logic if there exist a linearly ordered set $A$ and a function $f: V \times A \rightarrow L_{0}$ for which the following two conditions hold:

- For every $v \in V, a \in A$, and $b \in A$, if $a \leq b$, then $f(v, a) \leq f(v, b)$.

- For every $u, v \in V, u \leq v$ if and only if

$$
\forall a(f(u, a) \leq f(v, a))
$$

Theorem 3. A partially ordered set can be obtained by intervalization of classical logic if and only if it is linearly ordered.

Comment. In other words, an arbitrary partially ordered set can be obtained from the classical 2-valued logic by the following two-step procedure:

- first, we apply the intervalization to classical logic, and get linearly ordered sets $L$;

- then, we apply intervalization to linearly ordered sets $L$, and get all possible partially ordered sets.

\section{Proofs}

\section{A. Proof of Theorem 1}

We will start this proof by using the fact that, according to Zorn's Lemma, every set $V$ can be well-ordered, i.e., we can mark each element of this set by an ordinal number in such a way that different ordinal numbers correspond to different elements of the set. In other words, elements from $V$ are $v_{1}, v_{2}, \ldots, v_{\omega}$, etc. (In case the set is finite, this is simply numbering.) Let us fix one such numbering of the set $V$.

Based on this well-ordering of the set $V$, we can define a well-ordering of the set of all pairs $V \times V$ : namely, we define a lexicographic version of the well-ordering: $\left(v_{\alpha}, v_{\beta}\right)<\left(v_{\gamma}, v_{\delta}\right)$ if and only if either $\alpha<\gamma$, or $\alpha=\gamma$ and $\beta<\delta$.
We will construct the sets $A$ and $L$ and the function $f$ by using transfinite induction over these intervals. On each step of this construction, we will have a part $A^{\prime}$ of $A$ and a part $L^{\prime}$ of $L$, and the corresponding part of $f$ will map $V \times A^{\prime}$ into $L^{\prime}$. On each step, we will have the property that $f$ is non-strictly increasing in both variables, i.e.:

- if $a \leq b$, then $f(v, a) \leq f(v, b)$, and

- if $u \leq v$, then for every $a \in A^{\prime}$, we have $f(u, a) \leq$ $f(v, a)$.

We start with an element $v_{1} \in V$. For this element, we take a 1-element set $A^{\prime}=\left\{a_{0}\right\}$ and $L^{\prime}=\{0,1\}$. For this set, we define $f\left(v, a_{0}\right)$ as follows:

- if $v \leq v_{1}$, we take $f\left(v, a_{0}\right)=0$;

- otherwise, we take $f\left(v, a_{0}\right)=1$.

One can easily check that this function $f$ satisfies the two monotonicity conditions.

Let us now describe the induction step. Let us assume that we have already constructed sets $A^{\prime}$ and $L^{\prime}$, and that we have defined $f: V \times A^{\prime} \rightarrow L^{\prime}$. If on this set, for every pair $(u, v) \in V \times V$, we have

$$
u \leq v \leftrightarrow \forall a \in A^{\prime}(f(u, a) \leq f(v, a))
$$

then the theorem is proven. Assume now that there exist some pairs for which this condition is not satisfied. Let $\left(u^{\prime}, v^{\prime}\right)$ be the first pair, in lexicographic well-ordering of $V$, for which this condition is not satisfied. Since $f$ is monotonic in the second variable, this means that $u^{\prime} \not \leq v^{\prime}$, but $\forall a \in A^{\prime}\left(f\left(u^{\prime}, a\right) \leq f\left(v^{\prime}, a\right)\right)$. Let us expand $A^{\prime}, L^{\prime}$, and $f$ so as to cover this pair $\left(u^{\prime}, v^{\prime}\right)$.

Let us define the new linearly ordered set $A^{\prime \prime}$ by adding, to $A^{\prime}$, a new element $a_{\text {new }}$ which is larger than all elements of $A^{\prime}$. With this addition, the new set $A^{\prime \prime}$ is still linearly ordered.

To the set $L^{\prime}$, we add two new elements $l_{1}<l_{2}$ such that both are larger than all elements from $L^{\prime}$. Thus enlarged set $L^{\prime \prime}$ is still linearly ordered.

Now, we need to expand $f$ from $V \times A^{\prime}$ to $V \times A^{\prime \prime}$. Since the only new element of the set $A^{\prime \prime}$ is the element $a_{\text {new }}$, we must, therefore, define the values $f\left(v, a_{\text {new }}\right)$ for all $v \in V$. We defined these values as follows:

- if $v \geq u^{\prime}$, then $f\left(v, a_{\text {new }}\right)=l_{2}$;

- otherwise, $f\left(v, a_{\text {new }}\right)=l_{1}$.

From our choice of $l_{i}$ and $a_{\text {new }}$, we can conclude that thus expanded function remains monotonic in both variables. For $u^{\prime}$ and $v^{\prime}$, we have $f\left(a_{\text {new }}, u^{\prime}\right)=l_{2}$ and $f\left(a_{\text {new }}, v^{\prime}\right)=$ 
$l_{1}$. Here, $f\left(a_{\text {new }}, u^{\prime}\right)=l_{2} \not \leq f\left(a_{\text {new }}, v^{\prime}\right)=l_{1}$. Thus, for the expanded function $f, u^{\prime} \not \leq v^{\prime}$ and

$$
\neg \forall a \in A^{\prime \prime}\left(f\left(u^{\prime}, a\right) \leq f\left(v^{\prime}, a\right)\right),
$$

and hence,

$$
u^{\prime} \leq v^{\prime} \leftrightarrow \forall a \in A^{\prime \prime}\left(f\left(u^{\prime}, a\right) \leq f\left(v^{\prime}, a\right)\right) .
$$

One can easily check that if this equivalence holds on a certain step, it will hold forever. Thus, whichever pairs we covered by this equivalence stay covered. So, by transfinite induction, we can conclude that eventually, we will cover all such pairs, and thus, get an intervalization of the original partially ordered set. The theorem is proven.

\section{B. Proof of Theorem 2}

The set $L$ is a linearly ordered set and therefore, a linearly ordered lattice. Every linearly ordered lattice is known to be distributive, i.e., $a \wedge(b \vee c)=(a \wedge b) \vee(a \wedge c)$. Therefore, for every two elements $u, v$, and $w$, we will conclude that for every $a$,

$$
f(u \wedge(v \vee w), a)=f((u \wedge v) \vee(u \wedge w), a),
$$

hence

$$
f(u \wedge(v \vee w), a) \leq f((u \wedge v) \vee(u \wedge w), a)
$$

and

$$
f(u \wedge(v \vee w), a) \geq f((u \wedge v) \vee(u \wedge w), a)
$$

Since these two inequalities are true for every $a$, then from the second property of the lattice obtained by intervalization, we will conclude that

$$
u \wedge(v \vee w) \leq(u \wedge v) \vee(u \wedge w)
$$

and

$$
u \wedge(v \vee w) \geq(u \wedge v) \vee(u \wedge w),
$$

i.e., that

$$
u \wedge(v \vee w)=(u \wedge v) \vee(u \wedge w)
$$

and therefore, that $V$ is a distributive lattice. But it is known that there are non-distributive lattices: e.g., the lattice of all linear subspaces of a linear space in which $L_{1} \leq L_{2}$ if and only if $L_{1}$ is a subset of $L_{2}$. (This lattice is used in quantum logic.) Thus, not every lattice can be obtained by intervalization. The theorem is proven.

\section{Proof of Theorem 3}

Let us first show that every linearly ordered set $V$ can be obtained by intervalization of classical logic. Indeed, we can take $A=V$, and $f(v, a)=1$ if $v \geq a$ and $f(v, a)=$
0 otherwise. One can easily check that this function is non-strictly monotonic in $a$, and that $u \leq v$ if and only if $\forall a(f(u, a) \leq f(v, a))$.

Indeed, if $u \leq v$, then, enumerating all possible cases, we can conclude that $f(u, a) \leq f(v, a)$. Vice versa, if $f(u, a) \leq f(v, a)$ for all $a$, then this inequality is true also for $a=u$, for which $f(u, a)=1$. Hence, $f(v, u) \geq 1$, i.e., $f(v, u)=1$ and, by definition of the function $f$, we have $v \geq u$.

To complete the proof of the theorem, we must now show that any partial order which is obtained by intervalization of classical logic is linearly ordered. We will prove this fact by reduction to a contradiction. Let us assume that a partially ordered set $V$ which is not linearly ordered can be obtained by intervalization of classical logic. The fact that $V$ is not linearly ordered means that there exist two elements $u$ and $v$ for which $u \not \leq v$ and $v \not \leq u$. From the first inequality $u \leq \leq v$, we conclude that it is not true that $\forall a(f(u, a) \leq f(v, a)$; therefore, there exists an $a_{1}$ for which $f\left(u, a_{1}\right) \not \leq f\left(v, a_{1}\right)$. Since the function $f$ takes values in the set $L_{0}=\{0,1\}$, the only possibility of $f\left(u, a_{1}\right) \not \leq f\left(v, a_{1}\right)$ if when $f\left(u, a_{1}\right)=1$ and $f\left(v, a_{1}\right)=0$.

Similarly, from the fact that $v \not \leq u$, we conclude that there exists an element $a_{2} \in A$ for which $f\left(u, a_{2}\right)=0$ and $f\left(v, a_{2}\right)=1$.

The set $A$ is linearly ordered, so either $a_{1} \leq a_{2}$ or $a_{2} \leq$ $a_{1}$.

- In the first case $a_{1} \leq a_{2}$, we should have $f\left(u, a_{1}\right) \leq$ $f\left(u, a_{2}\right)$, which contradicts to the fact that $f\left(u, a_{1}\right)=$ 1 and $f\left(u, a_{2}\right)=0$.

- In the second case $a_{2} \leq a_{1}$, we get a similar contradiction between the conclusion $f\left(v, a_{2}\right) \leq f\left(v, a_{1}\right)$ and the fact that $f\left(v, a_{2}\right)=1$ and $f\left(v, a_{1}\right)=0$.

In both cases, we get a contradiction. Thus, our assumption is false, and the non-linearly ordered set $V$ cannot be obtained by intervalizing classical logic. The theorem is proven.

\section{ACKNowledgments}

This work was supported in part by NASA under cooperative agreement NCC5-209, by NSF grants No. EEC9322370 and DUE-9750858, by United Space Alliance, grant No. NAS 9-20000 (PWO C0C67713A6), by Future Aerospace Science and Technology Program (FAST) Center for Structural Integrity of Aerospace Systems, effort sponsored by the Air Force Office of Scientific Research, Air Force Materiel Command, USAF, under grant number F49620-95-1-0518, and by the National Security Agency under Grants No. MDA904-98-1-0561 and MDA904-98$1-0564$.

The authors are thankful to anonymous referees for their valuable comments. 


\section{REFERENCES}

[1] M. Mukaidono, "Interval truth values in fuzzy logic", Proceedings of the International Workshop on Breakthrough Opportunities for Fuzzy Logic, Tokyo Institute of Technology, 1996.

[2] M. Mukaidono, "Kleene algebras in fuzzy truth values", Proceedings of The Fourth International Workshop on Rough Sets, Fuzzy Sets, and Machine Discovery, The University of Tokyo, November 6-8, 1996, pp. 37-43.

[3] M. Mukaidono, "Algebraic structures of interval truth values in fuzzy logic", Proceedings IEEE-FUZZ'97, Barcelona, Spain, 1997.

[4] M. Mukaidono and H. Kikuchi, "Proposal on fuzzy interval logic", Japanese Journal of Fuzzy Theory and Systems, vol. 2, No. 2, pp. 99-117, 1990.

[5] M. Mukaidono, Y. Yam, and V. Kreinovich, "Intervals is all we need: an argument", Proceedings of The Eighth International Fuzzy Systems Association World Congress IFSA'99, Taipei, Taiwan, August 17-20, 1999, pp. 147-150.

[6] Y. Yam, M. Mukaidono, and V. Kreinovich, "Beyond $[0,1]$ to intervals and further: do we need all new fuzzy values?", Proceedings of The Eighth International Fuzzy Systems Association World Congress IFSA'99, Taipei, Taiwan, August 17-20, 1999, pp. $143-146$. 